\title{
ORIENTAÇÕES CONCETUAIS Dos EnFERMEIROS ESPECIALISTAS \\ Em Enfermagem De ReabilitaÇão Em Hospitais Portugueses
}

\author{
ORIENTACIONES ConCEPTUALES De Los ENFERMEROS EsPeCIALISTAS \\ En EnfERmería De ReHABilitación En Hospitales PoRTugueses
}

\section{Conceptual Guidelines Of Nurses Specialized In Rehabilitation Nursing In Portuguese Hospitals}

\author{
Maria Manuela Martins'; Olga Ribeiro²; João Ventura Da Silva ${ }^{3}$ \\ 1 - Escola Superior de Enfermagm do Porto - CINTESIS; 2 - Escola Superior de Saúde de Santa Maria - CINTESIS; 3 - CH de S. João
}

\section{RESUMO}

Objetivo: Identificar as conceções de enfermagem, pessoa, saúde e ambiente mais consensuais entre os enfermeiros especialistas em enfermagem de reabilitação.

Método: Estudo quantitativo, descritivo-exploratório, realizado em 36 instituições hospitalares, com a participação de 306 enfermeiros especialistas. Como instrumento de colheita de dados utilizou-se um questionário de autopreenchimento.

Resultados: Apesar de existirem diferenças nas cinco regiões do país, as conceções de enfermagem, pessoa, saúde e ambiente que os enfermeiros especialistas em enfermagem de reabilitação qualificam como totalmente de acordo com a sua prática são as de Afaf Meleis, Dorothea Orem, Callista Roy e Madeleine Leininger.

Conclusão: A congruência observada nos resultados relativamente às diferentes conceções revela a consistência da orientação concetual dos participantes. Neste sentido, o desafio passa por consolidar a sustentação do exercício profissional dos enfermeiros especialistas em enfermagem de reabilitação nos referenciais teóricos, cujas conceções são consideradas como totalmente de acordo com a sua prática.

Descritores: Enfermagem; Reabilitação; Modelos de Enfermagem.

\section{RESUMEN}

Objetivo: Identificar las concepciones de enfermería, persona, salud y ambiente más consensuadas entre los enfermeros especialistas en enfermería de rehabilitación.

Método: Estudio cuantitativo, descriptivo-exploratorio que se realizó en 36 instituciones hospitalarias, con la participación de 306 enfermeros especialistas. Como instrumento de recogida de datos, se utilizó un cuestionario de auto relleno.

Resultados: Aunque existen diferencias en las cinco regiones del país, las concepciones de enfermería, persona, salud y ambiente que los enfermeros especialistas en enfermería de rehabilitación califican como totalmente de acuerdo con su práctica son las de Afaf Meleis, Dorothea Orem, Callista Roy y Madeleine Leininger.

Conclusión: La congruencia observada en los resultados con respecto a las diferentes concepciones revela la consistencia de la orientación conceptual de los participantes. En este sentido, el desafío pasa por consolidar la sustentación del ejercicio profesional de los enfermeros especialistas en enfermería de rehabilitación en los referenciales teóricos cuyas concepciones son consideradas como totalmente de acuerdo con su práctica.

Descriptores: Enfermería; Rehabilitación; Modelos de Enfermería.

\section{ABSTRACT}

Objective: To identify the most consensual conceptions of nursing, individuals, health and environment among the nurses specialists in rehabilitation nursing.

Method: A quantitative, descriptive-exploratory study was carried out in 36 hospital institutions, and 306 specialist nurses were involved in this research. As a tool for data collection, it was performed a self-completion questionnaire.

Results: Although there are differences in the five regions of the country, the nursing, individuals, health and environment conceptions that nurses in rehabilitation nursing designate as fully in accordance with their practice are those of Afaf Meleis, Dorothea Orem, Callista Roy and Madeleine Leininger.

Conclusion: The congruence observed in the results regarding the different conceptions shows the consistency of the conceptual guidelines of the participants. In this sense, the challenge is to consolidate the support of the professional practice of nurses who are specialists in rehabilitation nursing in the theoretical frameworks whose conceptions are considered fully in accordance with their practice.

Descriptors: Nursing; Rehabilitation; Nursing Models. 


\section{INTRODUÇÃO}

Atualmente, tem vindo a ser consensual que a Enfermagem não pode avançar no sentido de satisfazer o seu compromisso social, se não existir clareza a respeito das suas bases disciplinares. Isto porque, sem uma orientação disciplinar clara e uma base para guiar o exercício profissional, é fácil perder o rumo. Neste contexto, todos os enfermeiros são convidados a sustentar o seu exercício profissional nos referenciais da disciplina, que mesmo existindo na teoria permanecem afastados da prática. Caso contrário, os enfermeiros correrão o risco de retroceder a ótimos profissionais técnicos ${ }^{(1)}$. Sempre que possível, a teoria deve ter uma relevância global, abrangendo os domínios genéricos e especializados, ajudando assim os enfermeiros a se afirmarem, aplicarem e avaliarem o seu papel único no âmbito dos cuidados de saúde. De facto, embora não seja solução para tudo, a teoria tem potencial para melhorar a prática, uma vez que responde aos desafios de um ambiente clínico complexo e em constante evolução, sendo fundamental para a sustentação da decisão clínica, quer no âmbito genérico ou especializado(2).

A enfermagem de reabilitação é uma área da intervenção especializada da enfermagem que tem como foco de atenção a manutenção e promoção do bem-estar e da qualidade de vida e a recuperação da funcionalidade, através da promoção do autocuidado, da prevenção de complicações e da maximização das capacidades $^{(3)}$. Até ao momento atual, o percurso nesta área de especialização tem colocado os enfermeiros especialistas em enfermagem de reabilitação numa posição privilegiada, para responder de forma sistematizada e intencional aos desafios e tendências da reabilitação( ${ }^{(4)}$. Todavia, à semelhança do que acontece no domínio genérico da enfermagem, para sistematizar a assistência no âmbito da enfermagem de reabilitação, é necessário que exista uma linha de pensamento que fundamente a prática. Entre as linhas de pensamento que podem ser utilizadas, os referenciais teóricos de enfermagem revelam-se como os mais adequados ${ }^{(5-6)}$. Importa, contudo, ter em consideração que, para identificar as teorias que melhor fundamentem a prática de cuidados, é necessário que haja congruência entre os conceitos estabelecidos pelos modelos teóricos (enfermagem, pessoa, saúde e ambiente) e o contexto de trabalho dos enfermeiros ${ }^{(5)}$.

Assim, partindo do pressuposto de que o exercício profissional da enfermagem de reabilitação deveria estar sustentado nos referenciais teóricos, interessounos perceber que referenciais serão mais consensuais entre os enfermeiros especialistas em enfermagem de reabilitação. Neste sentido, integrado numa investigação mais ampla, realizada no contexto nacional: "Contextos da prática hospitalar $e$ conceções de enfermagem: olhares sobre o real da qualidade e o ideal da excelência no exercício profissional dos enfermeiros", este estudo visou identificar as conceções de enfermagem, pessoa, saúde e ambiente mais consensuais entre os enfermeiros especialistas em enfermagem de reabilitação no contexto hospitalar.

\section{MÉTODO}

Inserido numa abordagem quantitativa, o estudo realizado foi descritivo, de cariz exploratório. Apesar de inicialmente ter sido planeada a sua concretização em todas as instituições hospitalares, enquadradas no modelo de gestão de Entidade Pública Empresarial (EPE), que aquando da colheita de dados eram 38, pelo facto de duas instituiçõos não aceitarem participar, o estudo foi realizado em 36 instituições hospitalares EPE de Portugal continental. Tendo em consideração os princípios ético-legais, de modo a obter autorização para a realização do estudo, foi enviada a todas as instituições hospitalares uma carta, dirigida ao conselho de administração, dando a conhecer o estudo e solicitando a participação. Apesar do processo inerente às autorizações ter variado de instituição para instituição, o estudo foi aprovado pelas comissões de ética e respetivos conselhos de administração das 36 instituições hospitalares envolvidas.

$\mathrm{Na}$ impossibilidade de estudar a totalidade da população, foi constituída uma amostra. A técnica de amostragem usada foi não probabilística por conveniência. Foram definidos como critérios de inclusão "ser enfermeiro especialista/especializado em enfermagem de reabilitação" e "exercer a sua atividade profissional na instituição hospitalar num período de tempo igual ou superior a seis meses, nos departamentos de medicina e especialidades médicas, cirurgia e especialidades cirúrgicas ou unidades de cuidados intermédios e intensivos". Neste sentido, todos os enfermeiros especialistas/especializados em enfermagem de reabilitação que exerciam funções nos serviços onde foi autorizado o estudo e que aceitaram participar, foram incluídos na amostra, que ficou constituída por 306 enfermeiros especialistas. Depois de esclarecidos sobre os objetivos, bem como sobre os procedimentos inerentes à investigação, através de informação escrita disponibilizada no serviço ou por presença física do investigador, aos enfermeiros especialistas que aceitaram participar no estudo, foi solicitado que assinassem o consentimento informado, tendo sido garantida a confidencialidade e o anonimato na utilização e divulgação das informações obtidas.

Como instrumento de colheita de dados foi usado o questionário, constituído por duas partes: Parte I Caracterização do respondente e Parte II - Conceções dos enfermeiros. A validade de construto desta segunda parte assenta nas conceções de enfermagem, pessoa, saúde e ambiente de 13 teóricas de enfermagem: Florence Nightingale (FN), Virginia Henderson (VH), Dorothea Orem (DO), Hildegard Peplau (HP), Imogene King (IK), Callista Roy (CR), Betty Neuman (BN), Moyra Allen (MA), Martha Rogers (MR), Rosemarie Parse (RP), Madaleine Leininger (ML), Jean Watson (JW) e Afaf Meleis (AM). Aquando do preenchimento do questionário, foi pedido aos enfermeiros especialistas/especializados que 
expressassem a sua opinião sobre os enunciados de cada teórica de enfermagem, relativamente aos quatro conceitos metaparadigmáticos: enfermagem, pessoa, saúde e ambiente. A escala de respostas do tipo Likert variou entre 1 e 5 , sendo que 1 correspondia a "está totalmente em desacordo com a minha prática", 2 "está em desacordo com a minha prática", 3 "não tenho opinião", 4 "está de acordo com a minha prática" e 5 "está totalmente de acordo com a minha prática". A colheita de dados foi realizada entre os meses de julho de 2015 a março de 2016. Para o tratamento dos dados, utilizámos o programa estatístico, Statistical Package for the Social Sciences, versão 22.0.

\section{RESULTADOS}

Quanto ao perfil sociodemográfico e profissional dos participantes, verificámos que dos 306 enfermeiros especialistas/especializados em enfermagem de reabilitação, a maioria é do género feminino $(71,2 \%)$, com idade média de 38,4 anos e um desvio de padrão de 7,6, predominando o estado civil de casado/união de facto $(60,5 \%)$. No que concerne ao grau académico, a licenciatura é maioritária $(74,84 \%)$, seguindo-se o mestrado (24,84\%) e o doutoramento (0,32\%). Relativamente à distribuição dos enfermeiros especialistas/especializados de acordo com as regiões da administração regional de saúde a que pertencem as respetivas instituições hospitalares, 49,3\% são do Norte, $21,6 \%$ de Lisboa e Vale do Tejo, 20,6\% do Centro, 5,2\% do Algarve e 3,3\% do Alentejo. No que diz respeito ao contexto onde exercem funções, predominaram os serviços de medicina e especialidades médicas $(47,1 \%)$, cirurgia e especialidades cirúrgicas $(38,2 \%)$ e unidades de cuidados intermédios e intensivos $(14,7 \%)$. Em relação ao exercício profissional na área da especialidade, o tempo médio foi de 3,7 anos, com um desvio de padrão de 4,7 , sendo o mínimo de 0 anos e o máximo de 23 anos. 0 valor mínimo de 0 anos deve-se ao facto de 132 enfermeiros (43,1\%) com cursos de especialização em enfermagem de reabilitação não exercerem a sua atividade profissional na área de especialidade. 0 tempo médio de exercício profissional no atual serviço foi de 8,8 anos, com um desvio de padrão de 7,0, sendo o mínimo de 1 ano e o máximo de 32 anos.

Uma vez evidenciadas as principais características dos participantes do estudo, atendendo ao objetivo anteriormente formulado, debruçar-nos-emos, em seguida, nos resultados mais significativos relativamente à concordância expressa pelos enfermeiros especialistas/especializados sobre as conceções de enfermagem, pessoa, saúde e ambiente. Assim, no contexto nacional, no que se refere ao conceito de enfermagem, as conceções que segundo os enfermeiros especialistas/especializados em enfermagem de reabilitação estão totalmente de acordo com a sua prática são as conceções de Afaf Meleis $(36,3 \%)$, Dorothea Orem $(35,6 \%)$, Callista Roy (33,0\%), Madeleine Leininger (29,4\%), Virginia Henderson (24,8\%), Florence Nightingale (15,4\%), Jean Watson $(12,1 \%)$, Imogene King $(8,2 \%)$, Betty
Neuman (7,2\%), Martha Rogers (6,9\%), Hildegard Peplau (6,5\%), Rosemarie Parse (6,5\%) e Moyra Allen $(6,2 \%)$ (Gráfico 1).

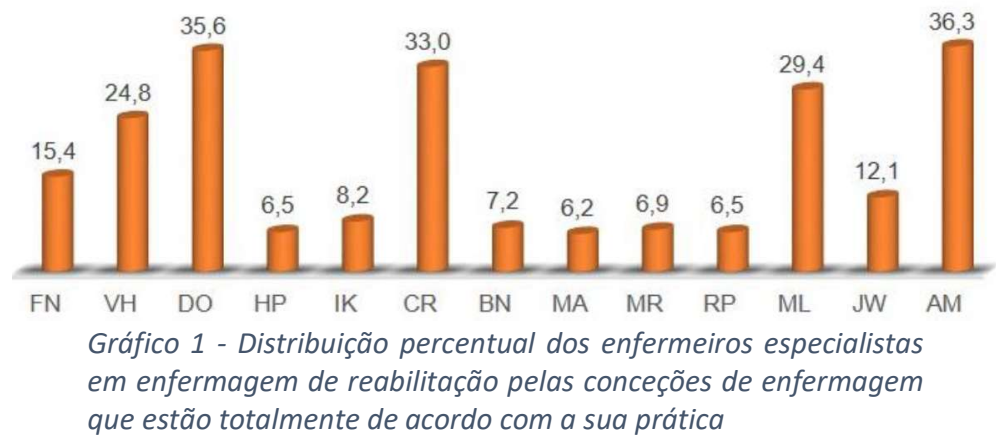

Relativamente ao conceito de pessoa, no contexto nacional, as conceções que segundo os enfermeiros especialistas/especializados em enfermagem de reabilitação estão totalmente de acordo com a sua prática são as conceções de Afaf Meleis $(44,1 \%)$, Dorothea Orem (35,0\%), Callista Roy (30,4\%), Madeleine Leininger $(27,5 \%)$, Virginia Henderson $(24,5 \%)$, Florence Nightingale $(14,4 \%)$, Jean Watson (9,8\%), Moyra Allen (8,8\%), Imogene King (8,2\%), Martha Rogers $(8,5 \%)$, Rosemarie Parse $(7,8 \%)$, Betty Neuman $(7,2 \%)$ e Hildegard Peplau $(7,2 \%)$ (Gráfico 2).

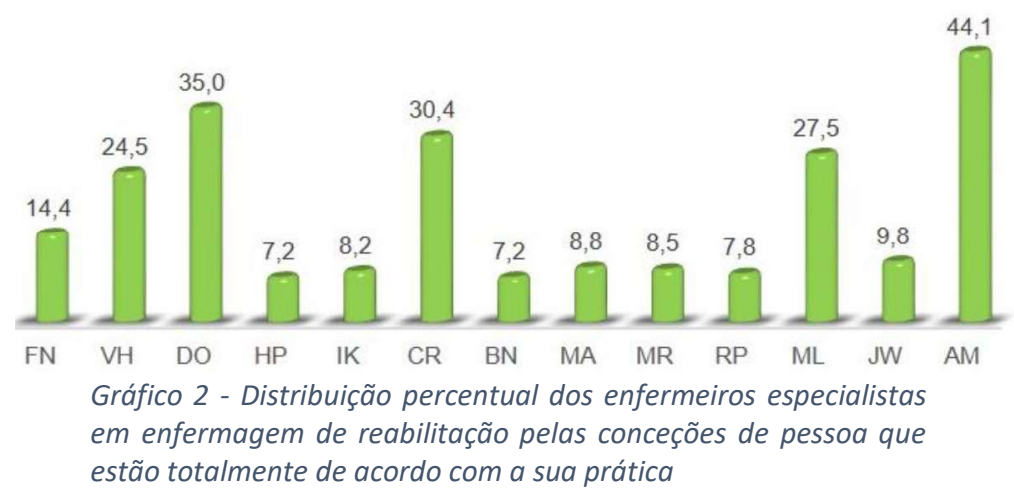

No que concerne ao conceito de saúde, no contexto nacional, as conceções que segundo os enfermeiros especialistas/especializados em enfermagem de reabilitação estão totalmente de acordo com a sua prática são as conceções de Afaf Meleis $(43,1 \%)$, Dorothea Orem (38,2\%), Callista Roy $(31,0 \%)$, Madeleine Leininger (27,8\%), Virginia Henderson (24,5\%), Jean Watson $(15,4 \%)$, Florence Nightingale $(13,1 \%)$, Martha Rogers $(8,2 \%)$, Hildegard Peplau $(7,8 \%)$, Imogene King (6,9\%), Moyra Allen $(7,2 \%)$, Rosemarie Parse $(6,9 \%)$ e Betty Neuman $(6,5 \%)$ (Gráfico 3).

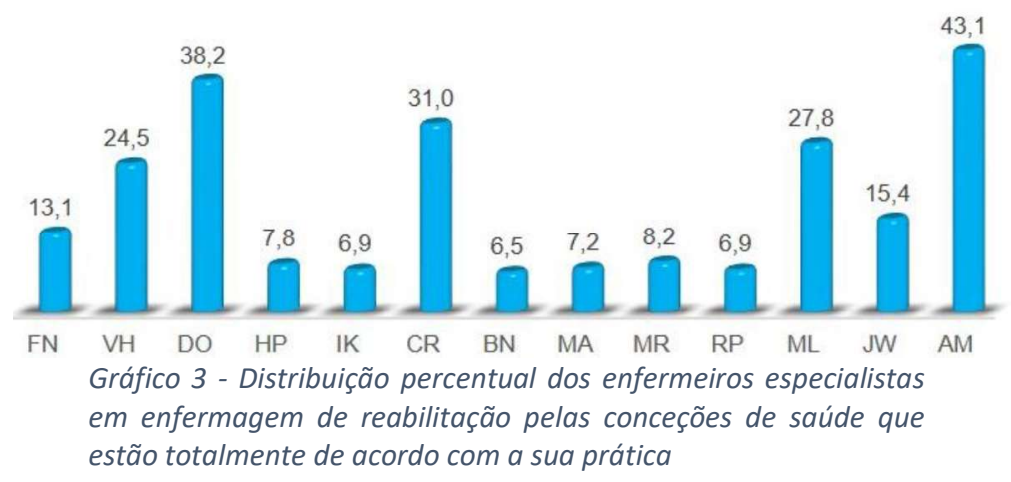


No que se refere ao conceito de ambiente, no contexto nacional, as conceções que segundo os enfermeiros especialistas/especializados em enfermagem de reabilitação estão totalmente de acordo com a sua prática são as conceções de Afaf Meleis $(41,8 \%)$, Dorothea Orem $(35,6 \%)$, Callista Roy (31,4\%), Madeleine Leininger (28,4\%), Virginia Henderson $(25,8 \%)$, Jean Watson $(15,4 \%)$, Florence Nightingale $(13,4 \%)$, Rosemarie Parse $(8,5 \%)$, Martha Rogers $(8,8 \%)$, Betty Neuman $(7,8 \%)$, Imogene King $(8,2 \%)$, Hildegard Peplau $(7,5 \%)$ e Moyra Allen $(6,2 \%)$ (Gráfico 4).

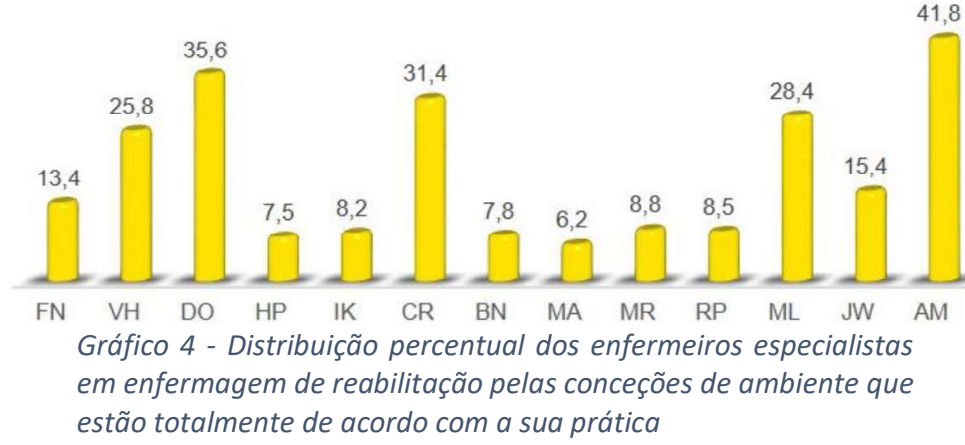

Após a análise da concordância dos enfermeiros especialistas/especializados em enfermagem de reabilitação relativamente às conceções de enfermagem, pessoa, saúde e ambiente, num contexto nacional, procedemos a uma análise regional. Para tal, as instituições hospitalares foram distribuídas pelas regiões das respetivas Administrações Regionais de Saúde, realizando-se, posteriormente, a comparação regional da distribuição das respostas. Para efeito de apresentação dos resultados, tal como anteriormente, continuam a salientar-se as conceções com que os enfermeiros especialistas/especializados em enfermagem de reabilitação mais se identificam.

A Figura 1 reflete as conceções de enfermagem que os enfermeiros especialistas/especializados qualificaram como totalmente de acordo com a sua prática. Analisando os resultados obtidos é interessante verificar que apesar da concordância com a conceção de enfermagem de Dorothea Orem ser transversal a todas as regiões do país, no Norte, Centro e Alentejo surgem com relevância as conceções de Afaf Meleis e Callista Roy, em Lisboa e Vale do Tejo as conceções de Madeleine Leininger e Afaf Meleis e no Algarve as outras conceções que se destacaram foram as de Madeleine Leininger e Callista Roy.

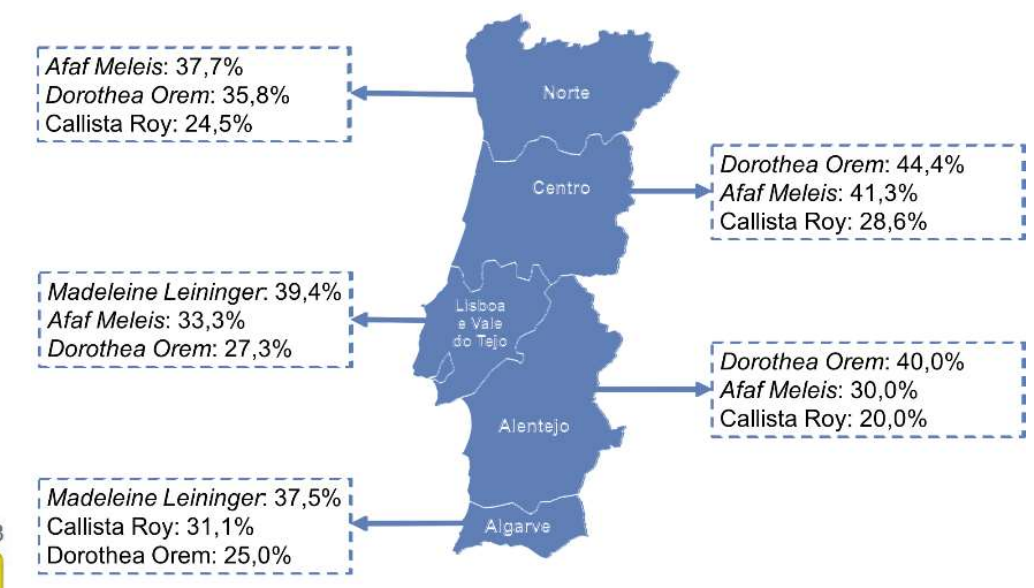

Figura 1 - Distribuição percentual, por regiões, relativa à concordância com as conceções de enfermagem

Ainda decorrente de uma análise por regiões, a Figura 2 reflete as conceções de pessoa que os enfermeiros especialistas/especializados qualificaram como totalmente de acordo com a sua prática. Numa análise aos resultados obtidos, verificou-se que apesar da concordância com a conceção de pessoa de Dorothea Orem ser transversal a todas as regiões do país, no Norte, Centro e Alentejo continuaram a surgir com relevância as conceções de Afaf Meleis e Callista Roy, em Lisboa e Vale do Tejo as conceções de Afaf Meleis e Madeleine Leininger e no Algarve as outras conceções que se destacaram foram as de Madeleine Leininger e Callista Roy.

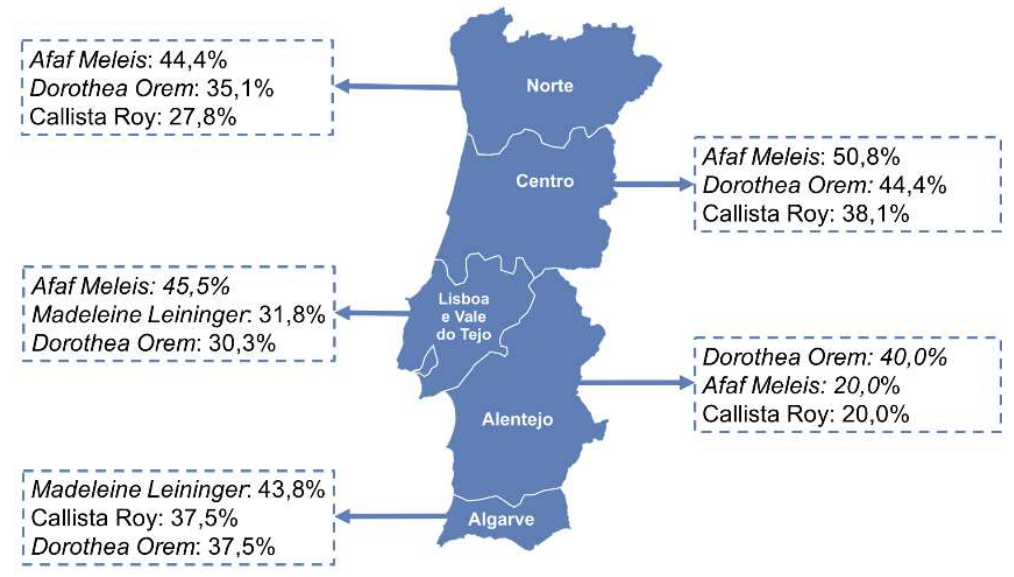

Figura 2 - Distribuição percentual, por regiões, relativa à concordância com as conceções de pessoa

Mantendo o enfoque numa análise por regiões, a Figura 3 reflete as conceções de saúde que os enfermeiros especialistas/especializados qualificaram como totalmente de acordo com a sua prática. Analisando os resultados obtidos, é interessante verificar que apesar da concordância com as conceções de saúde de Afaf Meleis e de Dorothea Orem serem transversais a todas as regiões do país, no Norte, Centro, Alentejo e Algarve surgiu com relevância a conceção de Callista Roy, e em Lisboa e Vale do Tejo a outra conceção que se destacou foi a de Madeleine Leininger. 


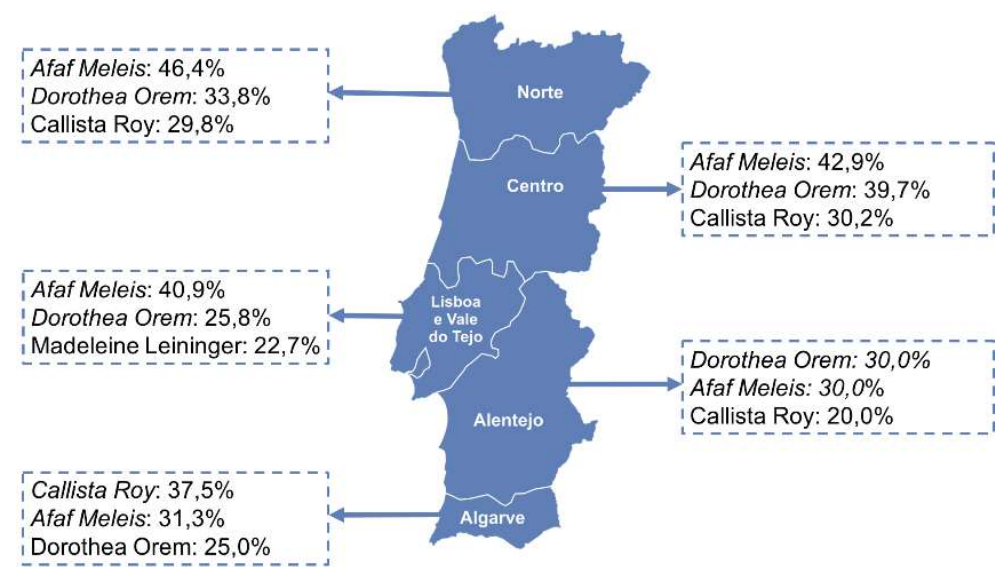

Figura 3 - Distribuição percentual, por regiões, relativa à concordância com as conceções de saúde

Ainda no âmbito de uma análise por regiões, a Figura 4 reflete as conceções de ambiente que os enfermeiros especialistas/especializados qualificaram como totalmente de acordo com a sua prática. Decorrente da análise aos resultados obtidos, constatou-se que apesar da concordância com as conceções de ambiente de Afaf Meleis e Dorothea Orem serem transversais a todas as regiões do país, no Norte, Centro, Alentejo e Algarve surgiu com relevância a conceção de Callista Roy, e em Lisboa e Vale do Tejo a outra conceção que se destacou foi a de Madeleine Leininger.

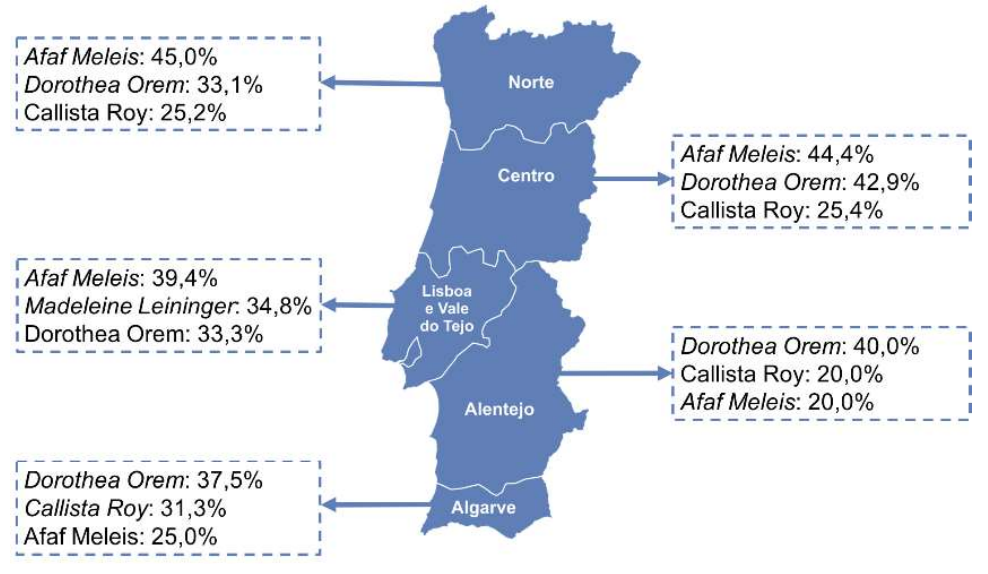

Figura 4 - Distribuição percentual, por regiões, relativa à concordância com as conceções de ambiente

Considerando os resultados enunciados relativamente aos quatro conceitos metaparadigmáticos, enfermagem, pessoa, saúde e ambiente, constatámos que no Norte, Centro e Alentejo é unânime a concordância com as conceções de Afaf Meleis, Dorothea Orem e Callista Roy. Em Lisboa e Vale do Tejo a concordância relativamente aos quatro conceitos verifica-se em relação às conceções de Afaf Meleis, Dorothea Orem e Madeleine Leininger. No Algarve a concordância verifica-se em relação às conceções de Dorothea Orem e Callista Roy (Figura 5).

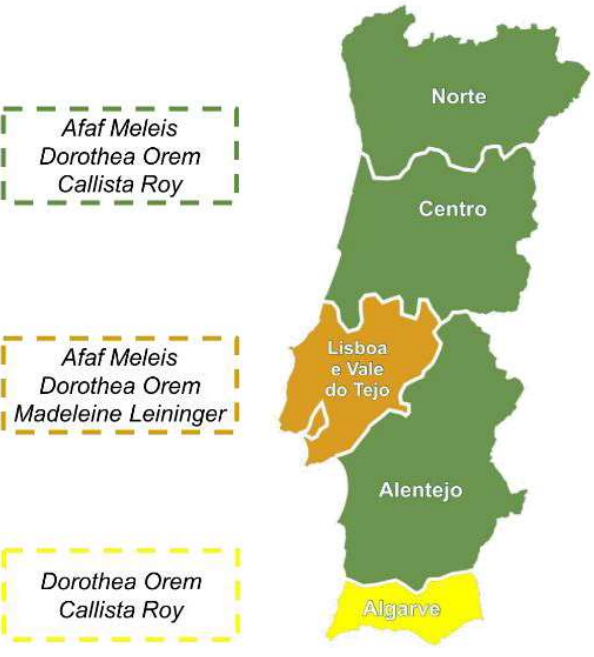

Figura 5 - Unanimidade na concordância com as conceções de enfermagem, pessoa, saúde e ambiente

\section{DISCUSSÃO}

$\mathrm{Na}$ sequência da análise das variáveis sociodemográficas e profissionais, verificámos que a maioria dos enfermeiros especialistas/especializados que participaram no estudo era do género feminino $(71,2 \%)$, tinha uma idade média de 38,4 anos e, predominantemente, o grau de licenciado $(74,84 \%)$. Para além destes resultados refletirem a realidade sociodemográfica dos profissionais de enfermagem em Portugal, vêm corroborar os dados atualizados pela Ordem dos Enfermeiros ${ }^{(7)}$ relativamente à área de especialização em enfermagem de reabilitação. Quanto ao tempo de exercício profissional na área da especialidade, embora oscilasse entre o mínimo de 0 anos e o máximo de 23 anos, o tempo médio foi de 3,7 anos. Salienta-se o facto de que $43,1 \%$ dos enfermeiros que participaram neste estudo, não exercerem a sua atividade profissional na área de especialidade, o que, mais uma vez, é revelador do não aproveitamento das qualificações dos enfermeiros ${ }^{(8)}$. Segundo dados publicados pela Ordem dos Enfermeiros, em dezembro de 2016, 46,8\% dos enfermeiros portugueses com especialização em enfermagem de reabilitação exerciam a sua atividade profissional no âmbito da prestação de cuidados gerais $^{(7)}$, o que vai ao encontro dos resultados obtidos neste estudo realizado no contexto nacional.

Embora nos documentos que sustentam o exercício profissional da enfermagem de reabilitação esteja explícito que na orientação da prática de cuidados de enfermagem de reabilitação, os modelos do autocuidado e das transições são estruturantes para a otimização da qualidade(3), este estudo permitiu-nos confirmar que atualmente os referidos modelos são parte integrante da orientação concetual dos enfermeiros especialistas em enfermagem de reabilitação. Efetivamente, os resultados obtidos neste estudo deixam claro que as conceções de enfermagem, pessoa, saúde e ambiente que os enfermeiros especialistas/especializados em enfermagem de reabilitação qualificam como totalmente de acordo com a sua prática são as de Afaf 
Meleis, Dorothea Orem, Callista Roy e Madeleine Leininger.

Apesar de não terem sido encontrados estudos que fornecessem dados numéricos sobre os modelos e as teorias de enfermagem mais utilizados pelos enfermeiros especialistas em enfermagem de reabilitação, a congruência observada nos resultados deixa claro que estes enfermeiros especialistas enfocam o seu papel na facilitação dos processos e das experiências humanas de transição; na promoção do autocuidado; nas capacidades de adaptação das pessoas, enfatizando a promoção da saúde, a estabilidade e a qualidade de vida, bem como nas atividades de assistir, apoiar e capacitar as pessoas a manter ou readquirir 0 bem-estar de formas culturalmente significativas.

À semelhança dos resultados obtidos num estudo realizado no contexto nacional com enfermeiros de cuidados gerais, enfermeiros especialistas e enfermeiros gestores ${ }^{(8-9)}$, é interessante verificar que a opção pelas conceções que mais se adequam à prática, seja maioritariamente a mesma, isto é, quando um enfermeiro especialista em enfermagem de reabilitação se identifica com um referencial teórico de enfermagem, identifica-se quase sempre, em relação aos quatro conceitos metaparadigmáticos: enfermagem, pessoa, saúde e ambiente. Nesse estudo anterior as conceções que obtiveram maior concordância foram as de Virginia Henderson, Afaf Meleis, Dorothea Orem, Madeleine Leininger e Callista Roy. Decorrente desses resultados, percebeu-se que, no âmbito dos cuidados gerais, é com base no referencial teórico de Virginia Henderson que os enfermeiros vão identificando as necessidades e os problemas dos clientes e, relativamente aos quais planeiam e executam as intervenções intencionalmente direcionadas para a substituição da pessoa naquilo que ela não pode fazer $^{(8-9)}$.

Atendendo a que no atual estudo, as conceções que obtiveram maior concordância foram as de Afaf Meleis, Dorothea Orem, Callista Roy e Madeleine Leininger, depreende-se que, perante a evolução da disciplina de enfermagem e da especialização em enfermagem de reabilitação, os enfermeiros especialistas têm vindo a empenhar-se em mudanças que julgam culminar numa prática sistematizada, e que portanto deve estar baseada nos pressupostos científicos e filosóficos que cada uma das teorias adotadas expõe ${ }^{(10)}$. As mudanças a que nos referimos estão principalmente relacionadas com 0 "afastamento" relativamente ao referencial teórico de Virginia Henderson e, consequentemente, com a apropriação dos referenciais de Afaf Meleis, Dorothea Orem e Callista Roy. Para além disso, comparativamente com os resultados do estudo já mencionado ${ }^{(8-9)}$, importa salientar que existe menor dispersão relativamente à concordância dos enfermeiros especialistas com as diferentes conceções. De facto, é visivelmente menor o valor percentual de enfermeiros especialistas/especializados que também considera as conceções de Florence Nightingale, Virginia Henderson, Hildegard Peplau, Imogene King, Betty
Neuman, Moyra Allen, Martha Rogers, Rosemarie Parse e Jean Watson, como totalmente de acordo com a sua prática.

Apesar de, empiricamente, no momento atual, termos conhecimento de que a tentativa de sustentar o exercício profissional nos referenciais teóricos está adstrita a alguns contextos, como resultado de iniciativas de alguns enfermeiros especialistas em enfermagem de reabilitação, importa relevá-la, uma vez que constitui uma oportunidade para a consolidação de um novo paradigma.

Este estudo veio fortalecer a ideia de que as teorias que têm vindo a moldar o exercício profissional dos enfermeiros especialistas em enfermagem de reabilitação são as de Afaf Meleis, Dorothea Orem, Callista Roy e Madeleine Leininger, sendo com base nesses referenciais teóricos que os enfermeiros vão validando as necessidades e os problemas reais e potenciais dos clientes e relativamente aos quais concebem e implementam planos de cuidados de enfermagem de reabilitação, tendo em vista melhorar as funções residuais, manter ou recuperar a independência, bem como minimizar o impacto das incapacidades instaladas, nomeadamente ao nível das funções neurológica, respiratória, cardíaca e/ou ortopédica $^{(11)}$.

Quanto aos resultados obtidos em relação aos referenciais teóricos que estão totalmente de acordo com a prática, são várias as justificativas possíveis. Por um lado, o investimento formativo efetuado nos últimos anos relativamente à pertinência dos referenciais teóricos de Afaf Meleis, Dorothea Orem, Callista Roy e Madeleine Leininger, no âmbito do exercício profissional dos enfermeiros especialistas em enfermagem de reabilitação, o que pode inclusive justificar as diferenças entre as regiões. Por outro, a confirmação pelas investigações realizadas, no contexto nacional e internacional, de que as conceções de Afaf Meleis, Dorothea Orem, Callista Roy e Madeleine Leininger são amplamente aplicáveis, sendo um contributo essencial na fundamentação e orientação da prática de enfermagem de reabilitação ${ }^{(4,12-14)}$. Na verdade, o recurso a teorias para orientar a prática contribuirá para uma atuação mais eficiente e efetiva, onde a colheita e organização dos dados, a formulação de diagnósticos, o planeamento e implementação das intervenções, bem como a definição de resultados, terão por base o mesmo fio condutor ${ }^{(6,9)}$.

\section{CONCLUSÃO}

A convicção de que o exercício profissional dos enfermeiros especialistas em enfermagem de reabilitação deveria estar sustentado nos referenciais teóricos e o desconhecimento de estudos centrados na identificação das orientações concetuais destes enfermeiros, constituíram as principais motivações para a realização deste estudo.

Em consonância com os resultados obtidos, verificámos que a opção pelas conceções que mais se adequam à prática, é maioritariamente a mesma, ou 
seja, quando um enfermeiro especialista se identifica com um referencial teórico, identifica-se quase sempre, em relação aos quatro conceitos metaparadigmáticos: enfermagem, pessoa, saúde e ambiente. Neste sentido, constatámos que os enfermeiros especialistas em enfermagem de reabilitação qualificaram como totalmente de acordo com a sua prática as conceções de Afaf Meleis, Dorotfhea Orem, Callista Roy e Madeleine Leininger.

Atendendo à relevância destes referenciais teóricos para a sustentação do exercício profissional dos enfermeiros especialistas em enfermagem de reabilitação, impõe-se como um desafio a sua incorporação nos contextos da prática, isto porque, apesar da evolução teoricamente significativa, só a consolidação dos fundamentos teóricos permitirá uma prática sistematizada e intencional.

Apesar dos contributos deste estudo, assumimos como limitação o facto da técnica de amostragem ter sido não probabilística, existindo a possibilidade do perfil de enfermeiros especialistas que participaram ter influenciado os resultados.

\section{REFERÊNCIAS}

1. Watson J. Elucidando a disciplina de enfermagem como fundamental para o desenvolvimento da enfermagem profissional. Texto \& Contexto Enferm. [Internet]. 2017 [cited 2018 Jun 24];26(4):editorial. Available from: http://www.scielo.br/pdf/tce/v26n4/0104-0707-tce-26-040000.pdf

2. McCrae N. Whither Nursing Models? The value of nursing theory in the context of evidence-based practice and multidisciplinary health care. J Adv Nurs. [Internet]. 2011 [cited 2018 Jun 26];68(1):222-229. Available from: https://www.ncbi.nlm.nih.gov/pubmed/21906131

3. Portugal. Ministério da Saúde. Regulamento n. ${ }^{\circ} 350 / 2015$, de 22 de junho de 2015. Regulamento dos Padrões de Qualidade dos Cuidados Especializados em Enfermagem em Enfermagem de Reabilitação. Diário da República. 2015 jun. 22; 2. ${ }^{a}$ série. p 16655-16660. Available from: https://www.ordemenfermeiros.pt/arquivo/legislacao/Docum ents/LegislacaoOE/RegulamentoPadQualidadeCuidEspecializEn fReabilitacao_DRJun2015.pdf

4. Andrade LT, Favoretto NB, Souza DRP, Gimenes FRE, Faleiros F. Diagnósticos, resultados e intervenções de enfermagem para indivíduos com lesão medular. In: NANDA International, Inc.; Herdman TH, Napoleão AA, Lopes CT, Silva VM, organizadoras. PRONANDA Programa de Atualização em Diagnósticos de Enfermagem: Ciclo 6. Porto Alegre: Artmed Panamericana; 2018. p. 9-45.
5. Carpinteira SF, Sanchez MC, Pereira MJ, Castro MR. The theoretical models in nursing services in vision of nurses of assistance: an exploratory study. ACC CIETNA [Internet]. 2014 [cited 2018 Jun 29];2(1):69-83. Available from: http://repositorio.usat.edu.pe/bitstream/usat/50/4/Acc\%20C ietna $\% 20$ Vol2\%20N\%C2\%B01\%20P7.pdf

6. McEwen M, Wills EM. Bases Teóricas de Enfermagem. $4^{\mathrm{a} e d .}$ Porto Alegre (RS): Artmed; 2016.

7. Ordem dos Enfermeiros. Membros ativos especialistas em reabilitação. Lisboa: Ordem dos Enfermeiros; 2016. Available from:

https://www.ordemenfermeiros.pt/arquivo/colegios/Docume nts/2017/DadosEstatisticos2016_Reabilitacao.pdf

8. Ribeiro OM. Contextos da Prática Hospitalar e Conceções de Enfermagem: Olhares sobre o real da qualidade e o ideal da excelência no exercício profissional dos enfermeiros. [Tese de Doutoramento]. Porto, Instituto de Ciências Biomédicas Abel Salazar, Universidade do Porto; 2017.

9. Ribeiro OM, Martins MM, Tronchin D, Forte, E. The pespective of portuguese nurses on nursing metaparadigmatica concepts. Texto \& Contexto Enferm. [Internet]. 2018 [cited 2018 July 17];27(2):e3970016. Available from: http://www.scielo.br/pdf/tce/v27n2/en_0104-0707-tce-2702-e3970016.pdf

10. Huitzi-Egilegor JX, Elorza-Puyadena MI, Urkia-Etxabe JM, Asurabarrena-Iraola C. Implementation of the nursing process in a health area: models and assessment structures used. Rev Latinoam Enferm. [Internet]. 2014 [cited 2018 Jun 29];22(5):772-777. Available from: https: //www.ncbi.nlm.nih.gov/pmc/articles/PMC4292680/

11. Portugal. Ministério da Saúde. Regulamento n. ${ }^{\circ} 125 / 2011$, de 18 de fevereiro de 2011. Regulamento das Competências Específicas do Enfermeiro Especialista em Enfermagem de Reabilitação. Diário da República. 2011 fev. 18; 2. ${ }^{a}$ série. p 8658-8659. Available from: https://www.ordemenfermeiros.pt/arquivo/legislacao/Docum ents/LegislacaoOE/Regulamento\%20125_2011_CompetenciasEs pecifEnfreabilitacao.pdf

12. Martins MM, Faria AC, Ribeiro OM, Monteiro MC, Reis MG. A longa transição do cliente com AVC sustentada no referencial teórico de Meleis. In: Figueiredo NM, Machado WC, Martins MM. Reabilitação: Nômades em busca de sentido para o cuidado da pessoa com deficiência adquirida. $1^{\mathrm{a} e d}$. Curitiba: CRV; 2018. p.159-194.

13. Martins MM, Monteiro MC, Ribeiro OM, Faria AC, Ferreira MS. Centralidade no autocuidado da pessoa com tetraplegia à luz do referencial teórico de Orem. In: Figueiredo NM, Machado WC, Martins MM. Reabilitação: Nômades em busca de sentido para o cuidado da pessoa com deficiência adquirida. $1^{\mathrm{a}} \mathrm{ed}$. Curitiba: CRV; 2018. p.195-221.

14. Martins MM, Ribeiro OM, Monteiro MC, Faria AC, Silva JM. Olhares sobre os cuidados de enfermagem à pessoa com paraplegia: contributos do modelo de Callista Roy. In: Figueiredo NM, Machado WC, Martins MM. Reabilitação: Nômades em busca de sentido para o cuidado da pessoa com deficiência adquirida. $1^{\text {a }}$ ed. Curitiba: CRV; 2018. p.223-255. 